\title{
Clinical performance of fluorescence- based methods for detection of occlusal caries lesions in primary teeth
}

Laura Regina Antunes PONTES(a) Tatiane Fernandes NOVAES ${ }^{(b)}$ Bruna Lorena Pereira MORO(a) Mariana Minatel BRAGA(a) Fausto Medeiros MENDES(a)

(a) Universidade de São Paulo - USP, School of Dentistry, Department of Orthodontics and Pediatric Dentistry, São Paulo, SP, Brazil.

(b) Universidade Cruzeiro do Sul, School of Dentistry, São Paulo, SP, Brazil.

Declaration of Interest: The authors certify that they have no commercial or associative interest that represents a conflict of interest in connection with the manuscript.

Corresponding Author: Fausto Medeiros Mendes E-mail: fmmendes@usp.br

https://doi.org/10.1590/1807-3107BOR-2017.vol31.0091

Submitted: May 11, 2017

Accepted for publication: Aug 28, 2017

Last revision: Sep 25, 2017
Abstract: We aimed to investigate the performance of fluorescencebased methods (FBMs), compared to visual inspection after histological validation, in detecting and assessing the activity status of occlusal carious lesions in primary teeth. One examiner evaluated 50 primary molars close to exfoliation in 24 children. Teeth were assessed using quantitative light-induced fluorescence (QLF) and pen-type laser fluorescence (LFpen). After exfoliation, histological validation was performed. Teeth were cut and sections were evaluated for lesion depth and activity status (after utilization of a $\mathrm{pH}$ indicator) under a stereomicroscope. Parameters related to the performance of the methods in detecting caries lesions at two thresholds (initial and dentin lesions) were calculated. Regarding the activity status, lesions were classified into sound+inactive or active, and the area under the ROC curve and the diagnostic odds ratio values of the methods were calculated and compared. Evaluation of red fluorescence using QLF presented higher sensitivity but lower specificity than visual inspection in detecting dentin caries lesions. However, QLF considering different parameters and LFpen had similar performance to that obtained with visual inspection. Regarding activity assessment, all FBMs and visual inspection also presented similar performance. In conclusion, FBMs did not prove advantageous for the detection and activity assessment of occlusal caries lesions in primary molars when compared to visual inspection.

Keywords: Dental Caries; Fluorescence; Diagnosis, Oral; Dental Caries Activity Tests; Tooth, Deciduous.

\section{Introduction}

Although visual inspection is the most widely used method for the detection and activity assessment of caries lesions ${ }^{1,2}$ it is subjective ${ }^{3}$ and it could be influenced by the examiners' experience. ${ }^{4}$ An attempt to overcome these limitations is the creation of detailed visual indices, such as the International Caries Detection and Assessment System (ICDAS). ${ }^{5}$ Actually, the use of detailed and validated indices improves the performance of visual inspection for the detection of carious lesions. ${ }^{2}$ Another advantage of visual inspection is that it permits the evaluation of activity status of carious lesions through validated additional criteria. ${ }^{6}$ However, even when well-described criteria are used, the method is examiner-dependent. 
Therefore, objective and quantitative tools, such as fluorescence-based methods (FBMs), have been increasingly researched for these purposes. ${ }^{3,7,8}$

One of these FBMs is diode laser fluorescence (LF), the first method launched in the market to detect occlusal carious lesions. ${ }^{7,9}$ After that, a pen-type laser fluorescence (LFpen) device was introduced for the detection of carious lesions on both occlusal and approximal surfaces. ${ }^{8}$ This device is based on a diode laser emitting a red light with a wavelength of approximately $655 \mathrm{~nm}$, at which the dental tissue emits fluorescence, indicated on a numerical scale from 0 to 99 . Higher values indicate more severe carious lesions. ${ }^{9}$ Even though these methods have been good at detecting occlusal and approximal caries lesions, ${ }^{8}$ they have not outperformed visual inspection. ${ }^{810}$

Another FBM is quantitative light-induced fluorescence (QLF), in which a blue light is emitted, capturing the back-scattered fluorescence in the green and red spectra. ${ }^{11}$ Loss of green fluorescence is observed in initial enamel carious lesions, and the QLF device translates this fluorescence into a quantitative value. Therefore, this method is appropriate for detecting the early signs of carious lesions and for monitoring the arrestment of these lesions after non-operative treatments, mainly on free smooth surfaces. ${ }^{3,8}$ However, studies to investigate the accuracy of QLF in detecting occlusal carious lesions have been unsatisfactory for permanent teeth, ${ }^{12,13}$ and no previous studies were conducted with primary teeth. Moreover, the accuracy of QLF in detecting occlusal caries lesions using histological evaluation has not been investigated as reference standard.

Furthermore, QLF is capable of detecting red fluorescence emitted by caries lesions, which is probably related to bacterial metabolic by-products. ${ }^{14,15}$ Thus, assessment of red fluorescence could be an adjunctive method for caries activity assessment, since active lesions are more infected than inactive ones. ${ }^{16,17}$ To the best of our knowledge, however, no previous study was carried out to evaluate the accuracy of QLF in detecting occlusal caries lesions, nor for the assessment of caries activity status in primary teeth.

Therefore, the objective of this clinical study with histological validation of the whole sample was to evaluate the accuracy of QLF and other methods in detecting severity and assessing the activity status of occlusal carious lesions in primary teeth, comparing FBMs with the visual inspection performed with ICDAS. The working hypothesis is that some of these FBMs are better at detecting the severity or assessing the activity status of caries lesions than visual inspection.

\section{Methodology}

\section{Ethical concerns and sampling}

The present study was approved by the local Research Ethics Committee (process number 203/2008). Guardians of the children who participated in the study signed an informed consent form prior to the study. This study used a sample from another recently published study. ${ }^{18}$ Children participating in this previous study who had at least one primary molar close to exfoliation were selected to participate in the present study.

For the first study, ${ }^{18}$ children who sought dental treatment in our dental school were randomly selected and invited to participate in the study through enrolment forms with no details on their oral health status. In the first study, 113 children aged 4 to 14 years were examined. ${ }^{18}$ Children who verbally agreed to their participation and whose parents signed the consent form were screened to check whether they had at least one primary molar close to exfoliation. Moreover, those eligible primary molars should not have enamel defects, restorations, sealants, or frankly cavitated lesions. Then, one suspect site (non-evident caries lesions) of each tooth included was chosen and marked on a drawing to indicate further evaluations. Therefore, we included apparently sound occlusal surfaces, surfaces with probable non-cavitated caries lesions, or microcavitated lesions, which were non-obvious in the first evaluation. This choice was made by an independent examiner who did not participate in the subsequent assessments (LRAP). More than one tooth per children could be selected.

\section{Clinical examination}

Prior to the examinations, the participants were positioned in a dental unit under operating light and subjected to cleaning procedures using a rotating bristle brush and water. No prophylactic pastes were used to 
avoid possible influence on measurements made by FBMs. Then, one trained examiner (TFN) carried out all examinations using the different methods.

The teeth were first assessed through visual inspection. The examination was performed in cleaned teeth, under illumination, with the aid of plane mouth mirrors and WHO periodontal probes. The drawing with the suspect site was shown to the examiner to guide the evaluation. The teeth were examined wet and were then dried for $5 \mathrm{~s}$ using a 3-in-1 syringe. The examiner evaluated the pre-selected site for the presence of lesions using the ICDAS. ${ }^{19}$ For activity assessment, the criteria described in the reference manual of the ICDAS, ${ }^{19}$ which is based on a previously described and validated system, ${ }^{20,21}$ were used. The examiner was instructed to classify the site according to the ICDAS scores, and in the presence of any type of carious lesion (scores from 1 to 6), the lesion was classified as active or inactive. The ICDAS and additional criteria used for activity assessment are described in Table 1.

\section{Fluorescence-based methods}

After the visual inspection, the LFpen method was used. This method was carried out using the DIAGNOdent pen (Kavo, Biberach, Germany), according to the manufacturer's instructions. Tip 2, appropriate for occlusal surfaces, was used. The device was first calibrated using the ceramic standard provided by the manufacturer, and also on a sound smooth surface of the tooth under evaluation. This value was electronically subtracted from those obtained from the examined site. After drying the tooth for $3 \mathrm{~s}$ with a 3-in-1 syringe, the tip was placed vertically and then rotated around the vertical axis. The examiner made two assessments on each suspicious site and recorded the mean value.

After that, the same examiner performed the evaluations with another FBM. The assessments were made with the QLF device (QLF Inspektor Pro; Inspektor Research Systems, Amsterdam, Netherlands), and the images were analyzed using a software program (Inspektor ProSoftware, Inspektor Research Systems, Amsterdam, Netherlands). The images were taken from the entire occlusal surface after drying with cotton pellets. Then, the marked area corresponding to the suspect site was manually selected and the images were assessed by the software. A sound region of the surface was defined as reference for the measurements. We recorded three different parameters provided by the QLF system:

a. Red fluorescence of the lesion $(\Delta \mathrm{R} \%)$ : percentage of ratio of red fluorescence compared with ratio of sound tissue;

b. Green fluorescence of the lesion $(\Delta \mathrm{F} \%)$ : percentage of green fluorescence loss on the suspicious site compared with green fluorescence from sound tooth tissue;

Table 1. International Caries Detection and Assessment System (ICDAS) ${ }^{5}$ and additional criteria used in the caries activity assessment. ${ }^{19,20,21}$

\begin{tabular}{|c|c|c|c|}
\hline Scores & ICDAS & Clinical features of active carious lesion & Clinical features of inactive caries lesion \\
\hline 0 & $\begin{array}{l}\text { No or slight change in enamel translucency } \\
\text { after prolonged air drying }(5 \mathrm{~s})\end{array}$ & - & - \\
\hline 2 & $\begin{array}{l}\text { First visual change in enamel (seen only } \\
\text { after prolonged air drying or restricted to } \\
\text { within the confines of a pit or fissure } \\
\text { Distinct visual changes in enamel } \\
\text { Localized enamel breakdown in } \\
\text { opaque or discoloured enamel (without } \\
\text { visual signs of dentinal involvement) }\end{array}$ & $\begin{array}{l}\text { Surface of enamel is whitish/yellowish } \\
\text { opaque with loss of luster; feels rough } \\
\text { when the tip of the probe is moved gently } \\
\text { across the surface. Lesion is in a plaque } \\
\text { stagnation area. }\end{array}$ & $\begin{array}{l}\text { Surface of enamel is whitish, brownish or } \\
\text { black. Enamel may be shiny and feels hard } \\
\text { and smooth when the tip of the probe is } \\
\text { moved gently across the surface. }\end{array}$ \\
\hline 4 & Underlying dark shadow from dentine & Probably active & - \\
\hline 6 & $\begin{array}{l}\text { Distinct cavity with visible dentin } \\
\text { Extensive distinct cavity with visible dentine } \\
\text { (involving more than half of the surface) }\end{array}$ & $\begin{array}{l}\text { Cavity feels soft or leathery on gently } \\
\text { probing the dentine. }\end{array}$ & $\begin{array}{l}\text { Cavity may be shiny and feels hard on } \\
\text { gently probing the dentine. }\end{array}$ \\
\hline
\end{tabular}


c. Green fluorescence loss integrated over the lesion area $\left(\Delta \mathrm{Q} \% . \mathrm{mm}^{2}\right)$ : percentage of green fluorescence loss in the lesion integrated over the lesion area.

\section{Reference standard}

Subsequently, teeth exfoliated or they were extracted (for orthodontic purposes) within 3 weeks after the end of the clinical recordings. The teeth were then stored at $-20^{\circ} \mathrm{C}$ for up to 1 month. ${ }^{22}$ Thereafter, the teeth were sectioned from the sites marked on the drawing using a 0.3-mm-thick diamond saw mounted in a cutting machine (Isomet 5000 Linear Precision Saw, Buehler, Lake Bluff, USA). The histological evaluation after sectioning was the reference standard of the present study. After that, the sections were first evaluated under a stereomicroscope at X8-20 magnification and reflected light (Leica DM 750, Leica Microsystems, Heidelberg, Germany). Immediately afterwards, two different examiners (LRAP and FMM), unaware of the results obtained with the methods, evaluated the sections for lesion depth. The lesions were classified according to a 5-point scale: $\mathrm{D} 0=$ no caries lesions, D1 = caries lesion limited to the outer half of the enamel, D2 = caries lesion reaching the inner half of the enamel; D3 = outer half dentin caries lesion and, D4 = dentin caries lesion reaching the inner half of the dentin.

Immediately thereafter, a $0.1 \%$ methyl red solution (Aldrich, Milwaukee, USA) was dripped onto the sections. After $1 \mathrm{~min}$, the excess dye was removed with a filter paper and the sections were evaluated as described previously. The examiners then classified the lesions into inactive, when the sections were stained yellow, or active, when the lesions appeared red or reddish. 22,23 After some training using images from other studies, the examiners performed the evaluations independently, and in case of disagreements, a consensus was reached after a discussion between them.

\section{Statistical analysis}

Regarding histological examinations, interexaminer reproducibility before the consensus between the examiners was evaluated using weighted kappa for both lesion depth and activity assessment.
As to lesion depth, receiver operating characteristics (ROC) analyses were carried out to evaluate the validity of the FBMs for detecting carious lesions at two different thresholds: all lesions (D0 = sound vs. D1, D2, D3 and D4 = carious), and dentin carious lesions (D0, D1 and D2 = sound vs. D3 and D4 = carious). The best cutoff points were determined (highest sum of specificity and sensitivity rates) for each method at both thresholds, as well as the areas under the ROC curves (Az).

With these cutoff points, sensitivity, specificity, and accuracy (percentage of right diagnosis, considering sound and carious surfaces) were calculated at both thresholds. The same parameters were also calculated for visual inspection considering lesions higher than ICDAS score 0 as cutoff point at all lesion thresholds and higher than score 2 for the dentin lesion threshold. The values obtained for each method were compared by McNemar's test.

With regard to activity assessment, the performance of FBMs was calculated in terms of diagnostic odds ratio (DOR) using logistic regression and Az values, considering the values obtained for each method. By using the best cutoff point calculated in the ROC analysis, results were dichotomized into sound + inactive vs. active, and DOR and accuracy were calculated. The cluster nature of the data (more than 1 tooth per child) was taken into account. The level of significance was set at 5\%, and MedCalc 13.1.2.0 (MedCalc software bvba, Ostend, Belgium) and Stata 13.0 (StataCorp LP, College Station, USA) were used for the analyses.

\section{Results}

Our sample included 50 occlusal primary teeth from 24 children ( 12 male and 12 female) aged 8 to 12 years (mean $=10.2$; standard deviation $=1.1$ years).

From the 50 teeth, histological examination revealed that only four teeth were sound (D0). The distribution of the caries lesions in terms of lesion depth is shown in Table 2. As to the reproducibility of the histological depth evaluation obtained before the consensus, the weighted kappa value was 0.915 (95\% confidence interval $-95 \% \mathrm{CI}=0.824$ to 1.000$)$. From 46 teeth with lesions, 16 with enamel lesions and 9 with dentin caries lesions were classified as active 
after the utilization of methyl red (a total of 25 active carious lesions) and 20 enamel lesions and 1 dentin carious lesion were classified as inactive (21 inactive caries lesions). Interexaminer reliability in assessing the activity status after the use of the dye yielded a weighted kappa of 0.800 (95\%CI $=0.634$ to 0.966$)$.

Regarding the presence of caries lesions, at D1 threshold, visual inspection presented higher sensitivity, but with no statistically significant differences compared

Table 2. Distribution among scores obtained with the International Caries Detection and Assessment System (ICDAS) and caries lesions activity status and the lesions depth evaluated through histological assessment

\begin{tabular}{|c|c|c|c|c|c|c|c|}
\hline \multirow{2}{*}{$\begin{array}{l}\text { ICDAS } \\
\text { scores }\end{array}$} & \multirow{2}{*}{$\begin{array}{l}\text { Activity } \\
\text { status }\end{array}$} & \multicolumn{5}{|c|}{ Lesion depth } & \multirow{2}{*}{ Total } \\
\hline & & D0 & D1 & D2 & D3 & D4 & \\
\hline 0 & Sound & 2 & 3 & 3 & & & 8 \\
\hline \multirow[t]{2}{*}{1} & Inactive & 2 & 3 & 12 & 1 & & 18 \\
\hline & Active & & 3 & 5 & 1 & & 9 \\
\hline \multirow[t]{2}{*}{2} & Inactive & & & 1 & 1 & & 2 \\
\hline & Active & & 3 & 1 & & 1 & 5 \\
\hline \multirow[t]{2}{*}{3} & Inactive & & & & & & 0 \\
\hline & Active & & & 1 & 1 & & 2 \\
\hline \multirow[t]{2}{*}{4} & Inactive & & & & & & 0 \\
\hline & Active & & & 1 & 4 & & 5 \\
\hline \multirow[t]{2}{*}{5} & Inactive & & & & & 1 & 0 \\
\hline & Active & & & & & & 1 \\
\hline \multirow{2}{*}{\multicolumn{2}{|c|}{ Total }} & 4 & 12 & 24 & 8 & 2 & 50 \\
\hline & & (8.0\% & $24.0 \%$ & $48.0 \%$ & $16.0 \%$ & $4.0 \%$ & $100.0 \%$ \\
\hline
\end{tabular}

D0: sound; D1: initial enamel caries lesions; D2: advanced enamel caries lesions; D3: initial dentin caries lesions; D4: advanced dentin caries lesions with the sensitivities obtained with LFpen and QLF considering $\Delta \mathrm{R}$ and $\Delta \mathrm{Q}$. The $\Delta \mathrm{F}$ obtained with QLF presented statistically significant lower sensitivity (Table 3). Both specificity and Az yielded similar results. However, regarding accuracy, visual inspection showed statistically significant and higher values than the $\Delta \mathrm{R}$ and $\Delta \mathrm{F}$ obtained with QLF (Table 3).

At the dentin threshold, although visual inspection and LFpen methods presented lower values, there were no statistically significant differences among the methods. On the other hand, specificity was significantly different from that obtained with QLF considering $\Delta \mathrm{F}$. The other parameters obtained with QLF $(\Delta \mathrm{R}$ and $\Delta \mathrm{Q})$ and LFpen presented intermediate specificity values, with no statistical differences (Table 3). Concerning accuracy and Az, all methods showed similar values (Table 3).

When we compared the methods regarding caries activity assessment, there were no statistically significant differences among them. In the analyses made considering the values obtained with FBMs (quantitative variables), Az values ranged from 0.67 to 0.77 (Table 4). When we dichotomized the variables considering the best cutoff points calculated through ROC analysis, accuracy was around 0.72 for all methods. DOR ranged from 6.43 (obtained with QLF, $\Delta \mathrm{F}$ ) to 12.74 (with LFpen). However, there were no significant differences since the $95 \% \mathrm{CI}$ values overlapped (Table 4).

Table 3. Performance of methods for detecting occlusal caries lesions at different thresholds regarding the lesion depth.

\begin{tabular}{|c|c|c|c|c|c|}
\hline Methods & Cut-off points & Sensitivity & Specificity & Accuracy & $\mathrm{Az}$ \\
\hline \multicolumn{6}{|l|}{ Initial enamel caries lesions (D1) } \\
\hline Visual inspection & $>0$ & $0.870^{\circ}$ & $0.500^{\circ}$ & $0.840^{a}$ & $0.766^{a}$ \\
\hline LFpen & $>4$ & $0.848^{\circ}$ & $0.500^{a}$ & $0.820 a, b$ & 0.649 a \\
\hline \multicolumn{6}{|l|}{ QLF } \\
\hline Red fluorescence $(\triangle R)$ & $>21.9$ & $0.717^{a, b}$ & $0.750^{\circ}$ & $0.720^{b, d}$ & $0.668^{\circ}$ \\
\hline Green fluorescence loss $(\Delta \mathrm{F})$ & $>9.3$ & $0.565^{b}$ & $0.750^{\circ}$ & $0.580^{c, d}$ & $0.590^{\circ}$ \\
\hline Green fluorescence loss $X$ lesion size $(\Delta Q)$ & $>2.1$ & $0.826^{\circ}$ & $0.500^{\circ}$ & $0.800 a, b$ & $0.571^{a}$ \\
\hline \multicolumn{6}{|l|}{ Dentin caries lesions (D3) } \\
\hline Visual inspection & $>2$ & $0.600^{\circ}$ & $0.950^{\circ}$ & $0.880^{a}$ & $0.871^{a}$ \\
\hline LFpen & $>30$ & $0.600^{\circ}$ & $0.825^{a, b}$ & $0.672^{a}$ & $0.674^{a}$ \\
\hline \multicolumn{6}{|l|}{ QLF } \\
\hline Red fluorescence $(\Delta \mathrm{R})$ & $>31.3$ & $0.900^{\circ}$ & $0.825^{a, b}$ & $0.840^{a}$ & $0.870^{a}$ \\
\hline Green fluorescence loss $(\Delta \mathrm{F})$ & $>16.7$ & $0.700^{\circ}$ & $0.875^{a, b}$ & $0.840^{a}$ & $0.780^{\circ}$ \\
\hline Green fluorescence loss $X$ lesion size $(\Delta Q)$ & $>13.8$ & $0.700^{\circ}$ & $0.775^{b}$ & $0.760^{\circ}$ & $0.745^{\circ}$ \\
\hline
\end{tabular}

Az: area under receiver operating characteristics curve. LFpen: pen-type laser fluorescence method; QLF: quantitative light-induced fluorescence method. Different letters indicate statistically significant differences among figures within the same column at the same threshold $(p<0.05)$. 
Table 4. Performance of visual inspection and fluorescence-based methods in assessing activity of occlusal caries lesions in primary teeth

\begin{tabular}{|c|c|c|c|c|c|}
\hline \multirow{2}{*}{ Methods } & \multicolumn{3}{|c|}{ Analysis considering quantitative values } & \multicolumn{2}{|c|}{$\begin{array}{l}\text { Analysis after dichotomization } \\
\text { considering the best cut-off points }\end{array}$} \\
\hline & $\begin{array}{c}\text { DOR } \\
(95 \% \mathrm{Cl})\end{array}$ & $\mathrm{Az}$ & Cut-off point & $\begin{array}{c}\text { DOR } \\
(95 \% \mathrm{Cl})\end{array}$ & Accuracy \\
\hline Visual inspection & * & * & * & $\begin{array}{c}8.78 \\
(2.12 \text { to } 36.45)\end{array}$ & 0.740 \\
\hline LFpen & $\begin{array}{c}1.04 \\
(1.01 \text { to } 1.08)\end{array}$ & 0.746 & $>8.5$ & $\begin{array}{c}12.74 \\
(2.46 \text { to } 66.12)\end{array}$ & 0.740 \\
\hline \multicolumn{6}{|l|}{ QLF } \\
\hline Red fluorescence $(\Delta R)$ & $\begin{array}{c}1.08 \\
(1.02 \text { to } 1.14)\end{array}$ & 0.766 & $>24.8$ & $\begin{array}{c}7.79 \\
(2.06 \text { to } 29.43)\end{array}$ & 0.740 \\
\hline Green fluorescence loss $(\Delta \mathrm{F})$ & $\begin{array}{c}1.08 \\
(0.98 \text { to } 1.19)\end{array}$ & 0.706 & $>9.28$ & $\begin{array}{c}6.43 \\
(1.72 \text { to } 24.05)\end{array}$ & 0.720 \\
\hline Green fluorescence loss $X$ lesion size $(\Delta Q)$ & $\begin{array}{c}1.01 \\
(0.99 \text { to } 1.03)\end{array}$ & 0.750 & $>3.53$ & $\begin{array}{c}11.23 \\
(2.33 \text { to } 54.10)\end{array}$ & 0.740 \\
\hline
\end{tabular}

DOR: diagnostic odds ratio; $95 \% \mathrm{Cl}: 95 \%$ confidence interval; $\mathrm{Az}$ : area under receiver operating characteristics. * Values were not calculated since visual inspection does not provide quantitative measures. We did not observe any statistical significant differences among the methods in any parameter.

\section{Discussion}

Caries diagnosis involves detection and activity assessment of caries lesions in order to choose the best treatment for the patients. ${ }^{20,21}$ This process is usually performed by visual inspection, ${ }^{3}$ which is the only validated method to assess caries activity ${ }^{6,}$ ${ }^{21,24}$ with high specificity for the detection of caries lesions. ${ }^{2}$ However, due to its subjective nature, quantitative methods have been suggested as possible alternatives. ${ }^{3,8}$ This study evaluated different FBMs for detecting and assessing the activity status of carious lesions in primary molars. This is the first study to investigate these methods, with later validation of all samples after exfoliation of primary teeth.

QLF has been extensively studied for quantification of small mineral changes after utilization of non-operative treatments in initial carious lesions, mainly in smooth surface caries., ${ }^{3,8,14}$ Nevertheless, the use of QLF for detecting occlusal carious lesions has not presented promising results in permanent teeth. ${ }^{12,13}$ With regard to the utilization of QLF for detecting initial lesions in primary teeth in our study, lower or similar sensitivity and accuracy values than those obtained with visual inspection were obtained with the different fluorescence parameters evaluated. Concerning specificity and Az, the values were similar among all methods.
As far as the accuracy in detecting dentin caries lesions is concerned, red fluorescence of the lesion assessed through QLF presented higher sensitivity than did visual inspection. Specificity, on the other hand, was lower than in visual inspection. Green fluorescence parameters presented similar values to those obtained with visual inspection. Although red fluorescence showed high sensitivity, other parameters, such as accuracy and Az, were higher in visual inspection. These findings observed for initial and dentin caries lesions corroborate the poor performance obtained with QLF for occlusal carious lesions in permanent teeth, as pointed out in previous studies. ${ }^{12,13}$

LFpen yielded similar results to those of visual inspection for initial lesions, but lower specificity for dentin caries lesions. We also observed great differences compared to the values obtained with red fluorescence in QLF. Although both methods are supposed to measure red fluorescence emitted by bacterial by-products, differences between the methods could explain these discrepancies. In LFpen, a diode laser emits a red light $(\lambda=655 \mathrm{~nm})$ through a sapphire tip, and the same tip collects the fluorescence emitted by the lesion. This fluorescence is indicated on a numerical scale from 0 to 99 . Higher values indicate more severe caries lesions. . $^{3,25,26}$

On the other hand, QLF is an intraoral camera that uses a high-intensity halogen lamp that emits 
a blue light $(\lambda=405 \mathrm{~nm})$ for excitation of the tooth. Then, the camera captures the fluorescence coming from the tooth (green fluorescence) and from bacterial metabolites (red fluorescence). A software program detects an increase of red fluorescence in the lesions and compares it with the red fluorescence emitted by a sound area of the same tooth. This difference between carious and sound sites are presented as percentage of increase of red fluorescence. ${ }^{3,8,11,14}$ Therefore, these differences between the two devices can explain the different performance observed in our study.

A limitation of our study is the small sample size, considering some parameters (for instance, specificity at all lesion thresholds and sensitivity at the dentin carious lesion threshold). This is so because it is difficult to obtain teeth close to exfoliation and also because the present study was nested in a study with a much larger sample. ${ }^{18}$ From 125 children and 606 teeth evaluated in the first study, ${ }_{18}^{18}$ we found 50 molars close to exfoliation that could be used in the present study.

However, some interesting findings can be seen in the present study. Sensitivity and specificity values obtained in the present study might be used in simulations, considering the prevalence obtained in studies with random or consecutive inclusion of participants. For instance, the improvement in sensitivity obtained with the red fluorescence parameter in QLF was 33\% and the decrease in specificity amounted to $13 \%$ compared to visual inspection. So, every 2.5 lesions correctly detected was followed by one sound surface incorrectly classified by QLF. However, if we consider a more realistic prevalence of non-evident occlusal caries lesions in the dentin, for example, $5.2 \%$, as obtained in an earlier study, ${ }_{10}^{10}$ one lesion detected by QLF corresponds to around seven sound surfaces classified as carious compared to visual inspection. Therefore, even if we consider the higher sensitivity obtained with some parameters assessed by the FBMs for detection of dentin carious lesions, this is not an advantage considering the low prevalence of these lesions in the current scenario of low caries prevalence. ${ }^{10}$

Another possible application of the FBMs investigated in our study is the possibility to evaluate the activity status of the lesions. Although the ICDAS criteria used in the present study presented predictive and construct validity when employed in primary ${ }^{6}$ and permanent ${ }^{27}$ teeth, a quantitative method could be useful for improving the reliability of this evaluation. Moreover, an objective method could be less influenced by examiner's experience than would visual inspection. ${ }^{4}$ Considering that LFpen and QLF (red fluorescence measurement) are methods based on the fluorescence emitted by microorganisms present in the caries lesions, ${ }^{9,11,15}$ and since active caries lesions are more infected than inactive ones, ${ }^{16}$ this possibility would be plausible. The old version of the LF method used with different drying times had been tested for this purpose previously, ${ }^{28}$ but this methodology was not compared with visual inspection.

In our study, however, regardless of the parameter compared (Az or DOR), the performance obtained with the FBMs did not present significant differences compared with that obtained with visual inspection. In a previous study, ${ }^{18}$ we observed that the red fluorescence measured by QLF was associated with the activity status evaluated by visual inspection (reference standard). However, other clinical parameters, such as the presence of visible biofilm, also had an association. On the other hand, a longitudinal retrospective study observed that the quantification of red fluorescence made by QLF could be useful in detecting lesions more prone to progress. ${ }^{16}$

The differences between our research and the previous study can be due to the different experimental designs. Our study evaluated criterion validity using a $\mathrm{pH}$ dye indicator, as in other studies published previously. ${ }^{22,23,28}$ Conversely, the other study retrospectively evaluated the progression of the lesions with higher red fluorescence using QLF; hence, the predictive validity of the method was assessed. ${ }^{16}$ This latter study ${ }^{16}$ actually has a superior design to evaluate the validity of methods for caries activity assessment. ${ }^{21}$ Nevertheless, as it is retrospective, ${ }^{16}$ and considering the fact that the caries activity assessment made by visual inspection also presented predictive validity using the same participants, ${ }^{24}$ visual inspection remains as a validated method for assessing the activity status of caries lesions that is easier to use and less expensive. Therefore, it should be indicated as the 
method for carious lesions activity assessment in daily clinical practice.

\section{Conclusions}

As FBMs did not present significant differences when compared to visual inspection, our hypothesis was not confirmed. Therefore, we can conclude that the FBMs do not present advantages over visual inspection for detecting and assessing the activity status of occlusal carious lesions in primary molars.

\section{References}

1. Bader JD, Shugars DA, Bonito AJ. A systematic review of the performance of methods for identifying carious lesions. J Public Health Dent. 2002;62(4):201-13. https://doi.org/10.1111/j.1752-7325.2002.tb03446.x

2. Gimenez T, Piovesan C, Braga MM, Raggio DP, Deery C, Ricketts DN, et al. Visual inspection for caries detection: a systematic review and meta-analysis. J Dent Res. 2015;94(7):895-904. https://doi.org/10.1177/0022034515586763

3. Braga MM, Mendes FM, Ekstrand KR. Detection activity assessment and diagnosis of dental caries lesions. Dent Clin North Am. 2010;54(3):479-93. https://doi.org/10.1016/i.cden.2010.03.006

4. Gimenez T, Bittar DG, Piovesan C, Guglielmi CAB, Fujimoto K, Matos R, et al. Influence of the examiners' experience on clinical performance of visual inspection in detecting and assessing the activity status of caries lesions. Oper Dent. 2013;38(6):583-90. https://doi.org/10.2341/12-067-C

5. Ismail Al, Sohn W, Tellez M, Amaya A, Sen A, Hasson H, et al. The International Caries Detection and Assessment System (ICDAS): an integrated system for measuring dental caries. Community Dent Oral Epidemiol. 2007;35(3):170-8. https://doi.org/10.1111/j.1600-0528.2007.00347.x

6. Guedes RS, Piovesan C, Ardenghi TM, Emmanuelli B, Braga MM, Ekstrand KR, et al. Validation of Visual Caries Activity Assessment: A 2-yr Cohort Study. J Dent Res. 2014; 93(7 Supp):101S-7S. https://doi.org/10.1177/0022034514531017

7. Bader JD, Shugars DA. A systematic review of the performance of a laser fluorescence device for detecting caries. J Am Dent Assoc. 2004;135(10):1413-26. https://doi.org/10.14219/jada.archive.2004.0051

8. Gimenez T, Braga MM, Raggio DP, Deery C, Ricketts DN, Mendes FM. Fluorescence-based methods for detecting caries lesions: systematic review, meta-analysis and sources of heterogeneity. PLoS One. 2013;8(4):e60421. https://doi.org/10.1371/journal.pone.0060421

\section{Acknowledgements}

The study was supported by the Conselho Nacional de Desenvolvimento Científico e Tecnológico (CNPq), Coordenadoria de Aperfeiçoamento de Pessoal de Nível Superior (CAPES), Fundação de Amparo à Pesquisa do Estado de São Paulo (FAPESP), and Pró-Reitoria de Pesquisa da USP. The authors would like to thank the participants of the Post-Graduation in Pediatric Dentistry Seminar of School of Dentistry, University of São Paulo (FOUSP) for their critical comments.
9. Hibst R, Paulus R, Lussi A. Detection of occlusal caries by laser fluorescence: Basic and Clinical investigations. Med Laser Appl. 2001;16(3):205-13. https://doi.org/10.1078/1615-1615-00024

10. Mendes FM, Novaes TF, Matos R, Bittar DG, Piovesan C, Gimenez T, et al. Radiographic and laser fluorescence methods have no benefits for detecting caries in primary teeth. Caries Res. 2012;46(6):536-43. https://doi.org/10.1159/000341189

11. Jong EJ, Higham SM, Smith PW, Daelen CJ, Veen MH. Quantified light-induced fluorescence, review of a diagnostic tool in prevention of oral disease. J Appl Phys. 2009;105(10):102031. https://doi.org/10.1063/1.3116138

12. Jallad $M$, Zero $D$, Eckert $G$, Ferreira Zandona $A$. In vitro detection of occlusal caries on permanent teeth by a visual, light-induced fluorescence and photothermal radiometry and modulated luminescence methods. Caries Res. 2015;49(5):523-30. https://doi.org/10.1159/000437214

13. Kühnisch J, Ifland S, Tranaeus S, Hickel R, Stösser $L$, Heinrich-Weltzien R. In vivo detection of non-cavitated caries lesions on occlusal surfaces by visual inspection and quantitative light-induced fluorescence. Acta Odontol Scand. 2007;65(3):183-8. https://doi.org/10.1080/00016350701291685

14. Heinrich-Weltzien R, Kuhnisch J, Veen M, Jong EJ, Stösser L. Quantitative light-induced fluorescence (QLF)--a potential method for the dental practitioner. Quintessence Int. 2003;34(3):181-8.

15. Bittar DG, Pontes LR, Calvo AF, Novaes TF, Braga MM, Freitas PM, et al. Is the red fluorescence of dental plaque related to its cariogenicity? J Biomed Opt. 2014;19(6):065004. https://doi.org/10.1117/1.JBO.19.6.065004

16. Felix Gomez G, Eckert GJ, Ferreira Zandona A. Orange/Red Fluorescence of Active Caries by Retrospective Quantitative Light-Induced Fluorescence Image Analysis. Caries Res. 2016;50(3):295-302. https://doi.org/10.1159/000441899 
17. Kim YS, Kang SM, Lee ES, Lee JH, Kim BR, Kim BI. Ecological changes in oral microcosm biofilm during maturation. J Biomed Opt. 2016;21(10):101409. https://doi.org/10.1117/1.JBO.21.10.101409

18. Novaes TF, Reyes A, Matos R, Antunes-Pontes LR, Marques RP, Braga MM, et al. Association between quantitative measures obtained using fluorescence-based methods and activity status of occlusal caries lesions in primary molars. Int J Paediatr Dent. 2017;27(3):154-62.

19. https://doi.org/10.1111/ipd.12242

20. International Caries Detection and Assessment System ICDAS. Coordinating Committee: criteria manual. Baltimore: International Caries Detection and Assessment System; 2005.

21. Nyvad B, Machiulskiene V, Baelum V. Reliability of a new caries diagnostic system differentiating between active and inactive caries lesions. Caries Res. 1999;33(4):252-60. https://doi.org/10.1159/000016526

22. Nyvad B, Machiulskiene V, Baelum V. Construct and predictive validity of clinical caries diagnostic criteria assessing lesion activity. J Dent Res. 2003;82(2):117-22. https://doi.org/10.1177/154405910308200208

23. Braga MM, Ekstrand KR, Martignon S, Imparato JC, Ricketts DN, Mendes FM. Clinical performance of two visual scoring systems in detecting and assessing activity status of occlusal caries in primary teeth. Caries Res. 2010;44(3):300-8. https://doi.org/10.1159/000315616
24. Ekstrand KR, Ricketts DNJ, Kidd EAM, Qvist V, Schou S. Detection, diagnosing, monitoring and logical treatment of occlusal caries in relation to lesion activity and severity: an in vivo examination with histological validation. Caries Res. 1998;32(4):247-54. https://doi.org/10.1159/000016460

25. Ferreira-Zandoná $A$, Santiago $E$, Eckert GJ, Katz BP, Pereira de Oliveira S, Capin OR, et al. The natural history of dental caries lesions: a 4 -year observational study. J Dent Res. 2012;91(9):841-6. https://doi.org/10.1177/0022034512455030

26. Lussi A, Hack A, Hug I, Heckenberger H, Megert B, Stich $H$. Detection of approximal caries with a new laser fluorescence device. Caries Res. 2006;40(2):97-103. https://doi.org/10.1159/000091054

27. Lussi A, Imwinkelried S, Pitts NB, Longbottom C, Reich E. Performance and reproducibility of a laser fluorescence system for detection of occlusal caries in vitro. Caries Res. 1999;33(4):261-6. https://doi.org/10.1159/000016527

28. Ferreira Zandoná A, Santiago E, Eckert G, Fontana M, Ando M, Zero DT. Use of ICDAS combined with quantitative light-induced fluorescence as a caries detection method. Caries Res. 2010;44(3):317-22. https://doi.org/10.1159/000317294

29. Braga MM, de Benedetto MS, Imparato JC, Mendes FM. New methodology to assess activity status of occlusal caries in primary teeth using laser fluorescence device. J Biomed Opt. 2010;15(4):047005. https://doi.org/10.1117/1.3463007 